See Article page XXX.

\section{Commentary: Brain damage during extracorporeal membrane oxygenation support: Looking where the light is!}

\author{
Keshava Rajagopal, MD, PhD, a,b \\ Faisal H. Cheema, MD, ${ }^{a, b, c}$ and Shuab Omer, MD $^{\text {b }}$
}

The idea that observations are constrained by the ability (or willingness) to observe is not new. As early as the 13th century, the Turkish Sufi satirist Nasreddin Hodga told the story of losing his ring in a room of his house. However, he proceeded to look for it in the courtyard. When asked by his wife why he would search for the ring in the courtyard, he replied that the room is dark, whereas the courtyard is well lit.

And so it is in many areas of investigation. In the context of short-term mechanical cardiac and/or pulmonary support, the only unqualified absolute contraindication is the absence of a viable neurological status (unless the support is in the context of bridging to organ donation), and central nervous system complications are the most devastating complications of therapy. But diagnoses may only be made when tests are performed. Although physical examination remains important in diagnosing neurological disorders, additional testing is typically required.

Ong and colleagues ${ }^{1}$ report the results of a study of diagnostic neuromonitoring undertaken in patients supported via extracorporeal membrane oxygenation (ECMO). Over a 64-month period, 215 patients underwent ECMO support. During the first 16 months, a standardized neuromonitoring protocol was not in place. In contrast, for the subsequent 48 months, a comprehensive protocol composed of

\footnotetext{
From the a Department of Clinical Sciences, University of Houston College of Medicine, Houston, Tex; ${ }^{\mathrm{b}}$ Houston Heart, HCA Houston Healthcare, Houston, Tex; and ${ }^{c}$ HCA Research Institute, Nashville, Tenn.

Disclosures: The authors reported no conflicts of interest.

The Journal policy requires editors and reviewers to disclose conflicts of interest and to decline handling or reviewing manuscripts for which they may have a conflict of interest. The editors and reviewers of this article have no conflicts of interest.

Received for publication Oct 12, 2021; revisions received Oct 12, 2021; accepted for publication Oct 13, 2021.

Address for reprints: Shuab Omer, MD, Houston Heart, HCA Houston Healthcare, 1200 Binz St, Suite 900, Houston, TX 77004 (E-mail: shueyb@yahoo.com).

J Thorac Cardiovasc Surg 2021; $\mathbf{\square}: 1-2$

$0022-5223 / \$ 36.00$

Copyright (c) 2021 Published by Elsevier Inc. on behalf of The American Association for Thoracic Surgery

https://doi.org/10.1016/j.jtcvs.2021.10.018
}

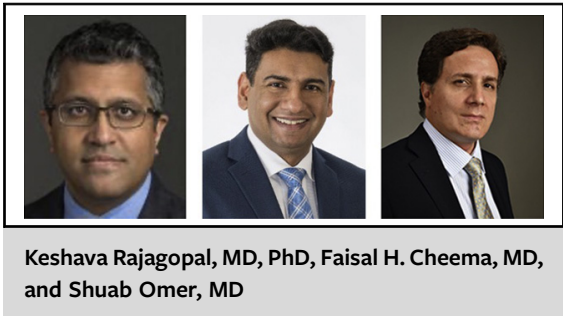

CENTRAL MESSAGE

Neuromonitoring in patients undergoing extracorporeal membrane oxygenation identifies acute brain injury, and this may facilitate expeditious treatment.

neocritical care consultation; serial neurological assessments in the absence of sedation; routine transcranial doppler studies; routine brain computerized tomography, electroencephalography if ECMO support was instituted in the setting of cardiac arrest, and/or Glasgow coma scale $<8$ or somatosensory evoked potentials if the Glasgow coma scale was $<4$; and routine postdecannulation brain magnetic resonance imaging was undertaken. The study found that-as expected-acute brain injury (ABI) was identified more commonly in the second era than in the first. However, more importantly, a dramatic improvement in the discharge neurological status was observed between the 2 eras. The authors suggest that this improvement may be attributable to enhanced ABI diagnosis resulting in more prompt and effective treatment. The 2 factors related to neurological outcomes, in a multivariable analysis, were the presence/absence of the neuromonitoring protocol and the presence/absence of pre-ECMO cardiac arrest.

There is, of course, no reason to think that the diagnostic studies were causally contributing to ABI. Rather, the investigators almost certainly observed an increased rate of $\mathrm{ABI}$ because they were shining a light by performing the studies in the first place. Yet, the improvement discharge neurological status is markedly disproportionate relative to the improvement in ABI diagnosis. That is rather difficult to explain, but may relate to patient population differences between the 2 eras (with the newer era having what appear to be better-selected patients, with lower incidences of preECMO cardiac arrest and use of venoarterial ECMO). Moreover, if indeed better diagnostics led to better treatment, this ought to be borne out by data relating to 
interventions consequent to $\mathrm{ABI}$ diagnosis. However, the study does not present such data.

Thus, it may be a stretch to suggest that improved neurological outcomes benefits were causally attributable to neuromonitoring. At the very least, improved diagnoses were achieved, and given the minimal risk of the assessments, it seems reasonable to suggest that neuromonitoring protocols ought to be instituted in patients supported by ECMO.

\section{Reference}

1. Ong CS, Etchill E, Dong J, et al. Neuromonitoring detects brain injury in patients on extracorporeal membrane oxygenation support. J Thorac Cardiovasc Surg. 2022. XX:XX-X. 